\title{
Substrate Effect on Plasmon Resonance of a Gold Nanoparticle Embedded Amorphous $\mathrm{BaTiO}_{3}$ Film
}

\author{
S. Ramakanth ${ }^{1}$, K. C. James Raju ${ }^{1,2^{*}}$ \\ ${ }^{1}$ Advanced Centre of Research in High Energy Materials (ACRHEM), University of Hyderabad, Hyderabad, India; ${ }^{2}$ School of Phys- \\ ics, University of Hyderabad, Hyderabad, India. \\ Email: *kcjrsp@uohyd.ernet.in
}

Received October $1^{\text {st }}, 2013$; revised November $9^{\text {th }}, 2013$; accepted November $16^{\text {th }}, 2013$

Copyright (C) 2013 S. Ramakanth, K. C. James Raju. This is an open access article distributed under the Creative Commons Attribution License, which permits unrestricted use, distribution, and reproduction in any medium, provided the original work is properly cited.

\begin{abstract}
Two sets of gold nanoparticles (NP) embedded in amorphous $\mathrm{BaTiO}_{3}$ films were prepared by sol-gel method using spin coating. Sample (1) is having $\mathrm{BaTiO}_{3}$ sol with $0.025 \mathrm{gm}$ of Chloroauric acid dissolved in $10 \mathrm{ml}$ of propan-2-ol, while sample (2) is having 0.086 gm of Chloroauric acid in the same amount of propan-2-ol. The films have been deposited on various substrates like borosilicate glass and fused silica. TEM images show that the particles are of 5 and $10 \mathrm{~nm}$ in size for the two set of samples, and some are having elongated morphology. Optical absorption properties of these films reveal the substrate and size effect on localised surface plasmon resonance (SPR). It shows a marginal red shift in the plasmon resonance peak from $414 \mathrm{~nm}$ to $420 \mathrm{~nm}$ in the case of sample (1) and $566 \mathrm{~nm}$ to $568 \mathrm{~nm}$ for sample (2) as the substrate changed from borosilicate glass to fused silica. It also shows red shift in Plasmon peak as the size increases from 5 to $10 \mathrm{~nm}$ and coincides with Mie explanation for the shift with size.
\end{abstract}

Keywords: Gold Nanoparticles; Gold NP Embedded Dielectrics; Substrate and Size Effect on Plasmon Resonance

\section{Introduction}

Recently, nanoparticles (NPs) attracted interest of research community because of their drastic change in various physical properties like conductivity, magnetism, optical properties etc. It has got many applications in various fields like optoelectronics, plasmonics, nanophotonics, and optically chemical sensors and in biology. The change in properties is the consequence of high surface to volume ratio, defects on the surface and geometry of the NPs [1]. The concept of nanoparticles interactions and its interaction with substrate, consequences of various shape emergences in synthesis are still an interesting problem to be solved theoretically. The SPR (Surface Plasmon Resonance) concept is widely used for different kind of sensors like explosive detection and bio sensor [2, $3]$. Gold NPs have been proved to be very useful in sensor applications [4]. The periodic patterns of Au NPs are very useful in $\mathrm{THz}$ generation which has application in explosive detection [4]. Also metal NP embedded dielectrics are of current interest in studying metal induced

"Corresponding author. crystallization. The study of capped nanoparticles with different chemicals is of interest in fundamental as well as in application point of view. This is not yet understood completely. One of such problems is the thiol capped gold NP. This gave a new physical property to gold NP which is unexpected for gold. The study of charge transfer effect in thiol capped gold NP gives the understanding of unexpected magnetism in gold [5]. Thus depending upon the bonding between the gold NP surface atoms, the capped chemical gives a variety of properties to gold NP. The charge transfer from gold NP to the dielectric matrix is very important in explaining unusual properties like magnetism and enhanced nonlinearity in gold NP embedded dielectric films. Apart from this the gold NP embedded dielectric films $\left(\mathrm{Au}\right.$ embedded $\left.\mathrm{TiO}_{2}\right)$ are very useful in understanding the laser induced damage mechanism [6]. Gold NP embedded $\mathrm{BaTiO}_{3}$ shows 10 times more enhanced third order optical nonlinearity $\left(\chi^{(3)}\right)$ compared to gold dispersed $\mathrm{SiO}_{2}$ films at $532 \mathrm{~nm}$ [7]. $\mathrm{BaTiO}_{3}$ also exhibits multifunctional properties in the nanoscale. Therefore studying the gold NP dispersed $\mathrm{BaTiO}_{3}$ is still interesting. The localised surface plasmon resonance is very sensitive to the host effects. Here we have studied 
the substrate effect on the localised surface plasmon resonance of gold NPs dispersed $\mathrm{BaTiO}_{3}$ film. The size and shape dependence of surface plasmon resonance of nanoparticles motivated the development of different chemical methods for the synthesis of metal nanoparticles. The control over the shape and size and inter particle distance of nanoparticles is very difficult in chemical methods. There are some colloidal methods which can give control over the size but not on interparticle separation.

\section{Experimental}

We have used sol-gel method to tune the size of gold nanoparticles embedded in $\mathrm{BaTiO}_{3}$ films. Barium acetate, Titanium isopropoxide and Chloroauric acid were the precursor chemicals for the method. Barium acetate was dissolved in $40 \mathrm{ml}$ of Propanoic acid and is stirred for 30 min. $2 \mathrm{ml}$ of Titanium isopropoxide was dissolved in 10 $\mathrm{ml}$ of Ethyl alcohol and added drop by drop to the dissolved Barium acetate in the presence of $\mathrm{N}_{2}$ atmosphere. This was again stirred for $3 \mathrm{hrs}$ [7]. Two kinds of samples were prepared by using different amount of Chloroauric acid. Sample (1) was prepared by mixing $0.025 \mathrm{gm}$ of Chloroauric acid containing propan-2-ol to $50 \mathrm{ml}$ of above prepared $\mathrm{BaTiO}_{3}$ sol. In the case of sample (2), the amount of Chloroauric was increased to $0.086 \mathrm{gm}$. This gold containing sol was used as coating solution. The films were deposited on borosilicate glass and fused silica substrates by spin coating (WS-400B-6NPP/LITE/8K) at $1000 \mathrm{rpm}$ for $5 \mathrm{sec}$ and $3000 \mathrm{rpm}$ for $5 \mathrm{sec}$. To get sufficient thickness we have deposited six layers of films in each sample case. The films were dried at $200^{\circ} \mathrm{C}$ for 2 $\mathrm{hr}$, and then heated to $400^{\circ} \mathrm{C}$ for $20 \mathrm{~min}$. The micro structural characterization is carried out by using, Tecnai 20 G2 STwin, FEI electron microscope, operated at $200 \mathrm{KV}$. The optical properties were carried out using JASCO V-570 UV/VIS/NIR spectrophotometer.

\section{Results and Discussion}

Here we have studied the substrate and particle size effect on localized surface plasmon resonance. It is an important technique in the detection of various microscopic organisms and absorbed chemicals including trace explosives. The organisms or chemicals which are to be detected when come in contact with gold NP, the dielectric constant of host changes. The change in dielectric constant of host will give the shift in surface plasmon resonance. Thus the shift in peak gives the preliminary information about the organisms and chemical. Normally the shift in wavelength corresponding to absorption peak is highly dependent on particle size, shape and interparticle spacing, when the nanoparticles are put inside the matrix and the film is supported by substrate. In this case the plasmon absorption peak is also sensitive to the di- electric constant of matrix and the substrate on which the NP containing film is deposited [8]. Mohammad et al. explained substrate and surrounding matrix effect on surface plasmon resonance by finite difference time domain simulation (FDTD) [9]. Here in this case the size and periodicity of particles is not well defined, hence the FDTD analysis will not help in analyzing the data of plasmon resonance.

Figures 1(a) and (b) show the TEM images of sample (1) on glass and fused silica substrate respectively. It is also clear that the gold NP's are of average $5 \mathrm{~nm}$ size. Similarly 1(c) and 1(d) shows for sample (2) that the average size of gold nanoparticles is $10 \mathrm{~nm}$ on glass and fused silica substrates respectively. The optical absorption spectrum in Figure 2 shows localised plasmon resonance peaks for sample (1) (2(a) on glass, 2(b) on fused silica) and for sample (2) (2(c) on glass, and 2(d) on fused silica). In the case of sample (1), the particles are of $5 \mathrm{~nm}$ in size and the plasmon resonance peak appears at $414 \mathrm{~nm}$. As the substrate changes to fused silica it shows a red shift in the absorption spectrum to $420 \mathrm{~nm}$. While in the case of sample (2), due to the increase in the Chloroauric acid, the particles are of $10 \mathrm{~nm}$ in size and show absorption at $566 \mathrm{~nm}$, and as the substrate changes to fused silica, it shows a small red shift to $568 \mathrm{~nm}$.

$$
Q(\lambda)=3 \pi \frac{N d^{3}}{\lambda} \frac{\varepsilon^{3 / 2}}{\ln (10)} \frac{\varepsilon_{i}}{\left(\left(\varepsilon_{r}+2 \varepsilon\right)^{2}+\varepsilon_{i}^{2}\right)}
$$

$\varepsilon_{r}=$ real part of dielectric constant. $\varepsilon_{i}=$ imaginary part of dielectric constant. $\varepsilon=$ dielectric constant of host medium. $d=$ diameter of nanoparticle.
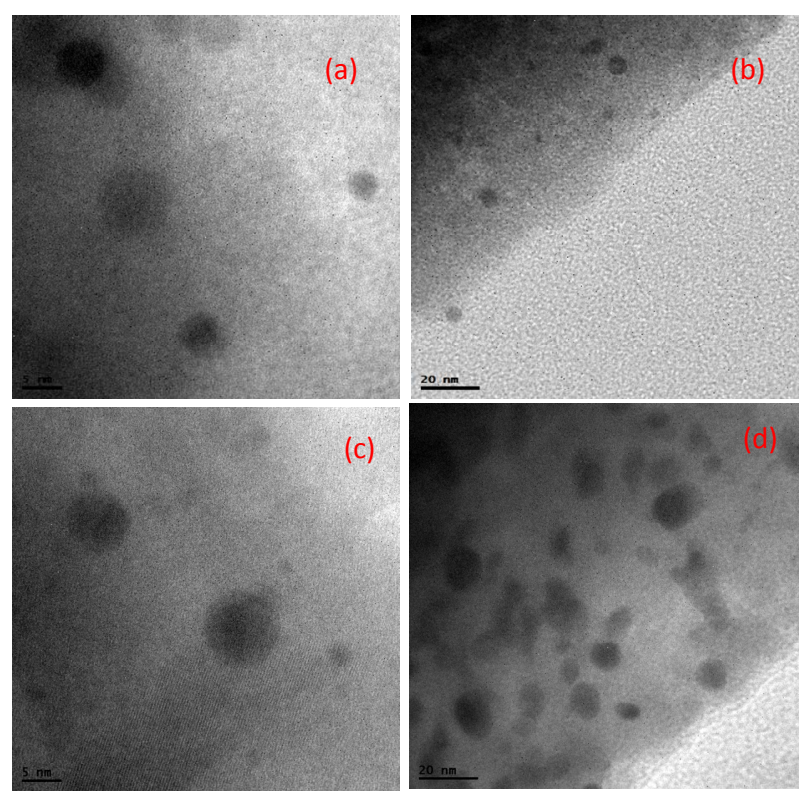

Figure 1. TEM image of gold NPs embedded in amorphous $\mathrm{BaTiO}_{3}$. Sample (1) (a) on glass; (b) on fused silica. Sample (2); (c) on glass; and (d) on fused silica. 
Thus, as discussed above the increase in Plasmon absorption peak wavelength with particle size increment is in agreement with Mie theory. The Equation (1) given above will give the relation between particle size, dielectric constant of host medium, dielectric constant of nanoparticle and scattering cross section. Table 1 summarizes the shift in Plasmon peak with particle size and with substrate. Hence, it is clear that localized plasmon resonance is sensitive to the substrate change. Both the particle size and substrate effect playing an important role in deciding the Plasmon peak of gold NP's in these films. The broad peak around $1000 \mathrm{~nm}$ may occur because of very small elongated gold NPs $\sim 2-3 \mathrm{~nm}$ in diameter $[10,11]$.

\section{Conclusion}

The understanding of gold-dielectric composite is of fundamental and technological importance. We successfully synthesized gold NP embedded in amorphous $\mathrm{BaTiO}_{3}$ film and studied the effect of substrate on localized surface plasmon resonance. The $5 \mathrm{~nm}$ Au NPs show a plasmon peak around $414 \mathrm{~nm}$ and particles with $10 \mathrm{~nm}$ show a peak around $566 \mathrm{~nm}$. As per Mie's theory it also shows red shift in Plasmon peak with increment in NP size. The films on the fused silica substrates show a red shift in the plasmon resonance peak compared to the films on the borosilicate glass substrates.

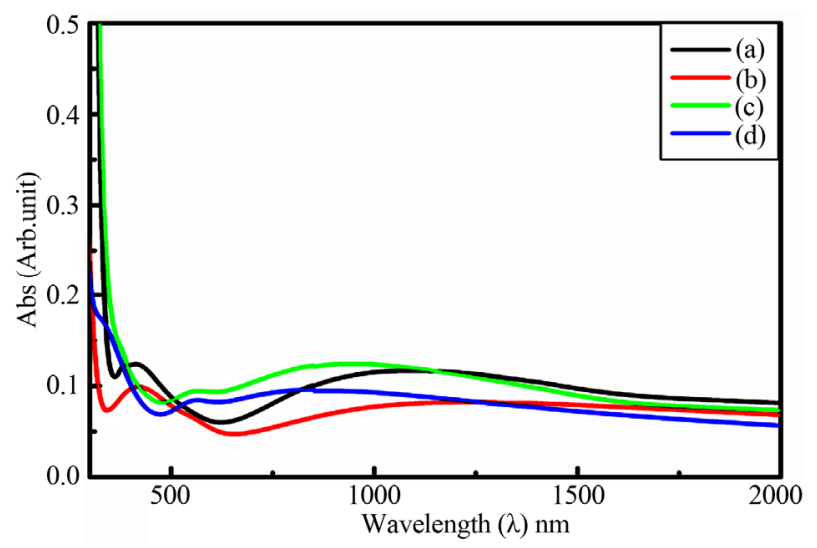

Figure 2. Absorption spectrum of gold NP embedded amorphous $\mathrm{BaTiO}_{3}$. Sample (1) (a) on glass; (b) on fused silica. Sample (2) (c) on glass; and (d) on fusedsilica.

Table 1. Plasmon absorption peak for 5 and $10 \mathrm{~nm}$ particles. Substrate and size effect on its plasmon resonance.

\begin{tabular}{cccccc}
\hline sample & \multicolumn{2}{c}{ 1 } & \multicolumn{2}{c}{2} \\
\hline NP size $(\mathrm{nm})$ & \multicolumn{2}{c}{5} & & \multicolumn{2}{c}{10} \\
substrate & Glass & FS & Glass & FS \\
Abs $(\lambda) \mathrm{nm}$ & 414 & 420 & 566 & 568 \\
\hline
\end{tabular}

\section{Acknowledgements}

We would like to thank DRDO, for their financial support, and Centre for Nano Technology at UOH for providing TEM based material characterizing facility.

\section{REFERENCES}

[1] F. Tam, A. L. Chen, J. Kundu, H. Wang and N. J. Halas, "Mesoscopic Nanoshells: Geometry-Dependent Plasmon Resonances beyond the Quasistatic Limit," Journal of Chemical Physics, Vol. 127, No. 20, 2007, Article ID: 204703. http://dx.doi.org/10.1063/1.2796169

[2] C. D Xiao and S. F. Sui, "Characterization of Surface Plasmon Resonance Biosensor," SENS ACTU-B, Vol. 66 No. 1-3, 2000, pp. 174-177. http://dx.doi.org/10.1016/S0925-4005(00)00372-5

[3] J. Zhao, X. Zhang, C. R. Yonzon, A. J. Haes and R. P. Vanduyne, "Localised Surface Plasmon Resonance Biosensors," Nanomedicine, Vol. 1, No. 2, 2006, pp. 219-228. http://dx.doi.org/10.2217/17435889.1.2.219

[4] Y. Gao, M.-Ku Chen, C.-En Yang, Y.-C. Chang, S. Yin, R. Hui, P. Ruffin, C. Brantley, E. Edwards and C. Luo, "Analysis of Terahertz Generation via Nanostructure Enhanced Plasmonic Excitations," Journal of Applied Physics, Vol. 106, No. 7, 2009, Article ID: 074302. http://dx.doi.org/10.1063/1.3236629

[5] P. Crespo, R. Litran, T. C. Rojas, M. Multigner, J. M. de la Fuente, J. C. Sanchez-Lopez, M. A. Garcia, A. Hernando, S. Penades and A. Fernandez, "Permanent Magnetism, Magnetic Anisotropy, and Hysteresis of Thiol-Capped Gold Nanoparticles," Physical Review Letters, Vol. 93, No. 8, 2004, Article ID: 087204-1-4. http://dx.doi.org/10.1103/PhysRevLett.93.087204

[6] S. Papernov, A. W. Schmid, R. Krishnan and L. Tsybeskov, "Laser-Induced Damage in Optical Materials," Vol. 4347, 2000, pp. 146-154.

[7] S. Otsuki, K. Nishio, T. Kineri, Y. Watanabe and T. Tsuchiya, "Optical Properties of Gold-Dispersed Barium Titanate Thin Films Prepared by Sol-Gel Processing," Journal of the American Ceramic Society, Vol. 82, No. 7, 1999, pp. 1676-1680.

http://dx.doi.org/10.1111/j.1151-2916.1999.tb01985.x

[8] G. Xu, Y. Chen, M. Tazawa and P. Jin, "Influence of Dielectric Properties of a Substrate upon Plasmon Resonance Spectrum of Supported Ag Nanoparticles," Applied Physics Letters, Vol. 88, No. 4, 2006, Article ID: 043114. http://dx.doi.org/10.1063/1.2167827

[9] M. A. Mahmoud, M. Chamanzar, A. Adibi and M. A. El$\mathrm{Sa}$, "Effect of the Dielectric Constant of the Surrounding Medium and the Substrate on the Surface Plasmon Resonance Spectrum and Sensitivity Factors of Highly Symmetric Systems: Silver Nanocubes," Journal of the American Chemical Society, Vol. 134, No. 14, 2012, pp. 6434-6442. http://dx.doi.org/10.1021/ja300901e

[10] R. Pasricha, A. Singh and M. Sastry, "Shape and Size Selective Separation of Gold Nanoclusters by Competitive Complexation with Octadecylamine Monolayers at the Air-Water Interface," Journal of Colloid and Interface 
Science, Vol. 333, No. 1, 2009, pp. 380-388. http://dx.doi.org/10.1016/j.jcis.2008.12.076

[11] S. K. Ghosh and T. Pal, "Interparticle Coupling Effect on the Surface Plasmon Resonance of Gold Nanoparticles:
From Theory to Applications," Chemical Reviews, Vol. 107, No. 11, 2007, pp. 4797-4862.

http://dx.doi.org/10.1021/cr0680282 